\title{
A Lateral Excitatory Network in the Escape Circuit of Crayfish
}

\author{
Jens Herberholz, Brian L. Antonsen, and Donald H. Edwards \\ Department of Biology, Georgia State University, Atlanta, Georgia 30302-4010
}

\begin{abstract}
A phasic stimulus directed to the rear of a crayfish (Procambarus clarkii) creates mechanosensory input to the lateral giant (LG) interneuron, a command neuron for escape. A single LG spike is necessary and sufficient to produce a highly stereotyped tail flip that thrusts the animal away from the source of stimulation. Here we describe a lateral excitatory network among primary afferent axons in the last abdominal ganglion of crayfish that produces nonlinear amplification of the sensory input to the command circuitry for escape. The lateral excitation is mediated by electrical synapses between central terminals of primary mechanosensory afferents. The network enables stimulated afferents to recruit unstimulated afferents that contribute additional input to $L G$ and to
\end{abstract}

mechanosensory interneurons that also converge on LG. When depolarized, the $L G$ neuron increases its own inputs from primary afferents and primary interneurons by facilitating the recruitment of both. Conversely, hyperpolarization of LG reduces the excitability of primary afferents and primary interneurons. The crayfish's decision to escape, previously thought to lie exclusively in the synaptic integrative properties of LG, is now seen to depend on the interactions between LG dendritic postsynaptic potentials and the responses of primary afferent terminals in the lateral excitatory network.

Key words: crayfish; escape; lateral giant neuron; afferents; interneurons; lateral excitation
Lateral inhibition among arrays of primary afferents or low-order interneurons is ubiquitous in sensory systems of vertebrates and invertebrates and serves to enhance spatial and temporal contrast (Hartline and Ratliff, 1957; Shepherd and Brayton, 1979). Much less understood are patterns of lateral excitation, in which the response of each afferent helps to excite neighboring afferents. Lateral excitation amplifies responses of neurons that are tuned to qualitatively similar stimuli and can be mediated by both chemical (Petersen and Sakmann, 2000) and electrical synapses (Hsu et al., 2000). Lateral electrical coupling occurs between the pedicles of primate retinal cones, where coupling between cones of the same spectral sensitivity enhances the signal-to-noise ratio, whereas coupling between cones of differing spectral type reduces it. Lateral electrical excitation also occurs among primary mechanosensory afferents involved in leg reflexes in locust (Burrows and Matheson, 1994) and crayfish (El Manira et al., 1993; Clarac et al., 2000), linking afferents that serve similar reflex functions, perhaps with the effect of increasing their combined input to a common set of postsynaptic cells.

We have found a lateral excitatory network among the mechanosensory afferents to the lateral giant (LG) interneuron in crayfish, a command neuron for tailflip escape (Edwards et al., 1999). Primary afferents innervate touch-sensitive hairs and stretch receptors in the tailfan (Wiese, 1976; Newland et al., 2001) and provide input to LG via monosynaptic and disynaptic pathways in the terminal ganglion (Krasne, 1969). The monosynaptic pathway is mediated primarily by rectifying electrical synapses, whereas the disynaptic pathway contains chemical synapses between primary afferents and mechanosensory interneurons and rectifying electrical synapses between the interneurons and LG (Zucker, 1972; Edwards et al., 1991, 1998; Newland et al., 2001).

Received May 17, 2002; revised July 24, 2002; accepted Aug. 15, 2002.

This work was supported by National Institutes of Health Grant NS26457.

Correspondence should be addressed to Jens Herberholz, Department of Biology, Georgia State University, 24 Peachtree Center Avenue Northeast, Atlanta, GA 30302-4010. E-mail: biojhh@panther.gsu.edu.

Copyright (C) 2002 Society for Neuroscience $\quad 0270-6474 / 02 / 229078-08 \$ 15.00 / 0$
The lateral excitatory network is mediated by patterns of electrical coupling among the terminals of primary afferents. It enables stimulated afferents to recruit unstimulated afferents; this recruitment is enhanced by depolarization of the LG neuron. Excitation of LG then depends on activation of a positive feedback between the depolarizing EPSP in LG and the recruitment of afferents that excite LG both directly and indirectly through mechanosensory interneurons.

\section{MATERIALS AND METHODS}

Crayfish (Procambarus clarkii) of both sexes and between 3.5 and $5 \mathrm{~cm}$ were obtained from commercial suppliers and kept in communal tanks for not $>2$ weeks before experiments. Animals were anesthetized on ice for 20-30 min, and the ventral nerve cords were exposed by removing the dorsal exoskeleton, viscera, and axial musculature. The saline used for all procedures was of the following composition (in $\mathrm{mM}$ ): $202 \mathrm{NaCl}, 5.37$ $\mathrm{KCl}, 13.53 \mathrm{CaCl}_{2}, 2.6 \mathrm{MgCl}_{2}$, and 2.4 HEPES, $\mathrm{pH}$ 7.4.

Anatomy. Dye coupling between LG and other members of the circuit was demonstrated by picospritzing (PV820 PicoPump; World Precision Instruments, Sarasota, FL) a mixture of $2 \%$ Neurobiotin (NB) (Vector Laboratories, Burlingame, CA) and 0.7\% 10,000 molecular weight (MW) dextran-linked Texas Red (TR) (Molecular Probes, Eugene, OR) in 0.3 $\mathrm{M} \mathrm{KCl}$ into the LG axon immediately rostral to A6 (Fig. 1). Desheathing of the A5-A6 connective was necessary to allow penetration of the low resistance (5-10 M $\Omega$ ) picospritzing electrodes. After filling the cell, the

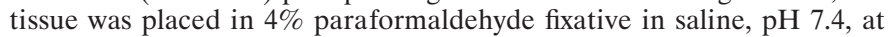
$4^{\circ} \mathrm{C}$ for $16 \mathrm{hr}$. After fixation, tissues were washed in three changes for 1 hr each of $0.1 \mathrm{M}$ phosphate buffer, $\mathrm{pH} 7.4$, with $0.1 \%$ Triton-X 100 (PBTX) at $4^{\circ} \mathrm{C}$, then placed in a 1:50 dilution of streptavidin-linked Alexa Fluor 488 (Molecular Probes) in PBTX for $16 \mathrm{hr}$ at $4^{\circ} \mathrm{C}$. After this, they were washed in four changes of PBTX for $1 \mathrm{hr}$ each and dehydrated. To visualize dye coupling between afferents, LG was filled via picospritzing $0.7 \%$ dextran-linked TR, and single afferents shown to be electrophysiologically connected to LG were filled with NB by iontophoresis. Lucifer Yellow (LY) (Sigma, St. Louis, MO) was iontophoresed into either LG or individual afferents in some preparations to confirm the NB dye-coupling results. All preparations were imaged on a Zeiss LSM 510 confocal microscope using $20 \times$ Fluor air interface and $63 \times$ $\mathrm{C}$-Apochromat water interface lenses. Full images of all labeled structures were acquired using the $20 \times$ objective and interslice intervals between 4 and $4.1 \mu \mathrm{m}$. Details of some preparations were imaged with the $63 \times$ objective and intervals of $2.0-2.1 \mu \mathrm{m}$. All images were captured using full resolution (2048X2048) and were stored with false colors based 


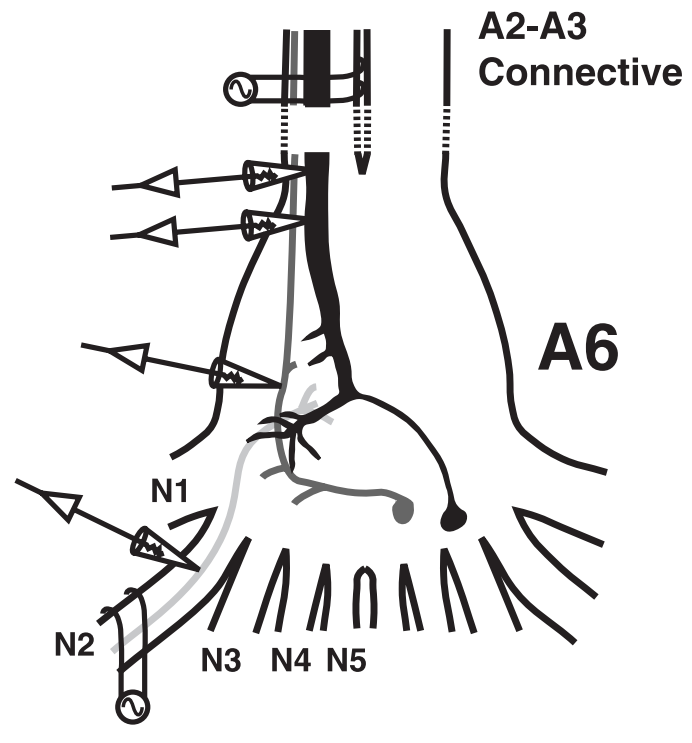

Figure 1. Schematic drawing of the crayfish's terminal ganglion (A6) and the peripheral sensory nerves $(N 1-N 5)$ in which all experiments were performed. The following parts of the LG escape circuit are shown: LG (black), primary mechanosensory interneuron (dark gray), and primary afferent (light gray). Extracellular and intracellular electrodes are shown in their typical positions.

on the emission of the fluorophores. Projections of confocal stacks were made using Adobe Photoshop 6 software with TIFF images exported from the Zeiss software.

Electrophysiology. Individual animals were firmly pinned in a Petri dish lined with Sylgard (Dow Corning, Midland, MI) and bathed in saline. One suction electrode was placed on the nerve cord rostral to the terminal ganglion to monitor the activity of ascending interneurons and to stimulate LG. One or two suction electrodes were placed on peripheral nerves of A6 to evoke afferent volleys (Fig. 1). Intracellular microelectrodes for recording and current injection had resistances of 15-35 $\mathrm{M} \Omega$. Because chloride-dependent depolarizing inhibition is ubiquitous in the escape circuit of crayfish (Edwards et al., 1991), we used 2 M potassium acetate-filled recording electrodes for afferents and interneurons to avoid changes in the chloride reversal potential that might convert inhibition to excitation. LG has a much larger volume, and recording microelectrodes filled with $3 \mathrm{M} \mathrm{KCl}$ proved to work best. LG was impaled with one or more microelectrodes in the initial segment in close proximity to A6 (Fig. 1) and identified by its response to sensory nerve stimulation. Afferent recording microelectrodes were placed in different sensory nerves close to their roots (Fig. 1). Primary afferents and mechanosensory interneurons, including Interneuron A, were identified by their response to nerve cord stimulation and by their morphology as revealed by injection with $\mathrm{NB}$ or LY. We used AxoClamp2A, AxoClamp2B (Axon Instruments, Foster City, CA) and a Getting microelectrode amplifier (model 5A) for current- and voltage-clamp experiments and a Grass stimulator (model S88) and an A-M Systems differential amplifier (model 1700) for stimulation and recording through suction electrodes. Clampex 8.0 and Clampfit 8.0 (Axon Instruments) were used to store and analyze the data.

Neuronal modeling. A multicompartment electrical circuit model was used to simulate responses and interactions in the lateral excitatory network. The model was built and tested using the program NEURON8J developed by Donald H. Edwards. The program uses a $5^{\text {th }}$-order RungeKutta-Feldberg numerical integration procedure. The reversal potentials for the three cell types were: afferents: $E_{\mathrm{Na}}=+45 \mathrm{~V}, E_{\mathrm{K}}=-70 \mathrm{mV}$, $E_{\mathrm{L}}=-75 \mathrm{mV}$; interneurons: $E_{\mathrm{Na}}=+45 \mathrm{mV}, E_{\mathrm{K}}=-65 \mathrm{mV}, E_{\mathrm{L}}=-78$ $\mathrm{mV}$; LG: $E_{\mathrm{Na}}=+45 \mathrm{mV}, E_{\mathrm{K}}=-70 \mathrm{mV}, E_{\mathrm{L}}=-78 \mathrm{mV}$. The corresponding maximal conductances were: afferents: $G_{\mathrm{Na}}=120 \mu \mathrm{S}, G_{\mathrm{K}}=36$ $\mu \mathrm{S}, G_{\mathrm{L}}=1 \mu \mathrm{S}$; interneurons: $G_{\mathrm{Na}}=160 \mu \mathrm{S}, G_{\mathrm{K}}=48 \mu \mathrm{S}, G_{\mathrm{L}}=1.33 \mu \mathrm{S}$; $\mathrm{LG}_{\mathrm{D}}$ (is passive): $G_{\mathrm{Na}}=0 \mu \mathrm{S}, G_{\mathrm{K}}=0 \mu \mathrm{S}, G_{\mathrm{L}}=1 \mu \mathrm{S} ; \mathrm{LG}_{\mathrm{IS}}$ (is active): $G_{\mathrm{Na}}=200 \mu \mathrm{S}, G_{\mathrm{K}}=60 \mu \mathrm{S}, G_{\mathrm{L}}=1.67 \mu \mathrm{S}$. Chemical synaptic current is described by $I_{\text {syn }}(t)=\left(V_{\text {post }}-E_{\text {syn }}\right) G_{\text {synmax }}\left(t / \tau_{\mathrm{s}}\right)^{\mathrm{s}} e^{-\mathrm{t} / \tau_{\mathrm{s}}}$, where $V_{\text {post }}$ is the postsynaptic membrane potential, $E_{\text {syn }}=0 \mathrm{mV}(\sim 70 \mathrm{mV}$ above rest potential), $G_{\text {synmax }}=0.15 \mu \mathrm{S}, s=0.5$, and $\tau_{\mathrm{s}}=1 \mathrm{msec}$. The rectifying
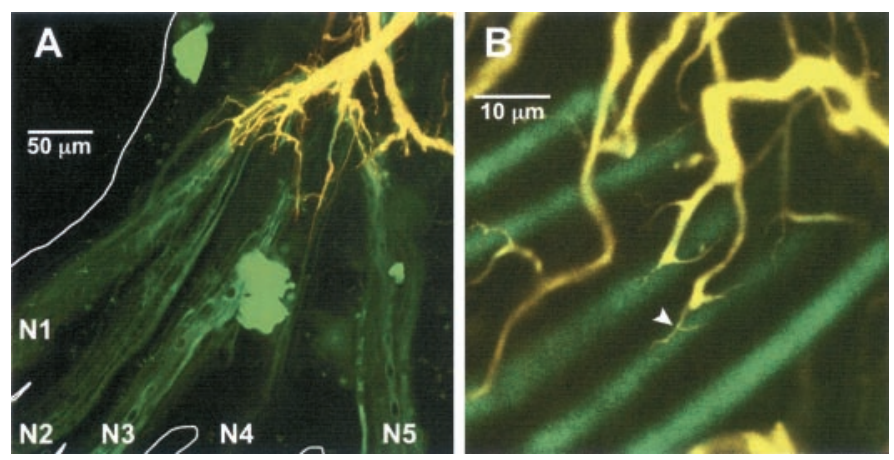

Figure 2. LG and neurons dye-coupled to LG in the terminal ganglion. LG was pressure-injected with two dyes, 10,000 MW dextran-linked TR and NB. A, Projected stack of confocal images of LG and dye-coupled afferents. The major yellow dendrite is the projecting LG; yellow results from superposition of the TR (red) and NB (green) fluorescence. The green axons that project out of the ganglionic nerves are primary afferent neurons; the green-stained somata are from dye-coupled motor neurons whose axons are out of the plane of focus. $B$, Single afferent axons dye coupled to terminal dendritic branches. Coupling appears to be mediated by the fine, vine-like dendritic processes that wrap around the cylindrical afferent axons (arrowhead). $A, B$, Results from two different experiments.

electrical synaptic conductances are described by $G_{\text {rec }}\left(V_{\text {pre }}-V_{\text {post }}\right)=$ $G_{\max }+\left(G_{\min }-G_{\max }\right) /\left(1+e^{(0.15 \times(V \text { pre }-V \text { post }))}\right)$, where $G_{\max }$ is 0.33 $\mu \mathrm{S}$ and $G_{\min }$ is $0.005 \mu \mathrm{S}$ (Giaume et al., 1987). The opening and closing time constants for the rectifying synapse conductance are assumed to be identical and equal to $\tau_{\text {rec }}=0.75 \mathrm{msec}$ (Jaslove and Brink, 1986). The kinetics of the conductance are given by the first-order differential equation $d G_{\mathrm{rec}}(t) / d t=\left[G_{\mathrm{rec}}\left(V_{\mathrm{pre}}-V_{\mathrm{post}}\right)-G_{\mathrm{rec}}(t)\right] / \tau_{\mathrm{rec}}$.

\section{RESULTS}

\section{Dye coupling}

The LG is dye-coupled to primary afferents (Zucker, 1972; Edwards et al., 1991, 1998; Antonsen and Edwards, 2000), as shown by passage of the low molecular weight tracer NB (367 MW) (Fig. 2 ). Dye coupling reveals that the afferent input from the tailfan is spatially segregated onto discrete areas of the dendritic tree of the LG (Fig. 2A). Each nerve innervates sensory organs on one region of the tailfan in an approximate lateromedial order $(\mathrm{Ca}-$ labrese, 1976), and this is reflected in their projection onto the LG dendritic tree. Furthermore, afferent-LG contacts occur at the distal terminals of each major dendritic branch (Fig. 2B). Injection of NB into axons of single primary afferents that monosynaptically excite LG reveals that each afferent is dye-coupled to between one and twelve other afferents (4.1 $\pm 0.9 ; N=29)$ (Fig. $3 A$ ). Distal to the contact point between the afferent axon and LG, the axon divides into an array of terminal branches that project into the ganglionic neuropil away from LG. These branches were not revealed by dye coupling from LG to the afferents, presumably because the dye is transported peripherally toward the cell somata. When injected into a single afferent axon, however, NB passed to other primary afferents through central contacts (Fig. $3 B$ ). Most $(92 \%)$ of the coupled afferents project into the ganglion through the same nerve as the injected afferent, but dye-coupled afferents that project in through other nerves are also found.

\section{Electrical coupling}

Stimulation of a single afferent evokes a small, phasic EPSP in LG as recorded in the initial segment (Fig. 3C); dendritic EPSPs are much larger (Zucker, 1972; Edwards et al., 1994, 1998). These synapses have been shown to be rectifying and voltage-dependent, but the rectification is partial (Edwards et al., 1991; Heitler et al., 
Figure 3. Coupling between primary afferents and between afferents and LG. $A$, Projected stack of confocal micrographs of LG, injected with TR alone, and primary afferents, one of which was injected with NB alone (solid arrow indicates the injection site). The NB spread into five other primary afferent axons. The contact point between the injected afferent and LG appears to occur at the tip of an LG dendrite (open arrow). Although the axons of the primary afferents appear to come together at this point in this projected stack, there is separation in the Z-plane. $B$, Enlargement of boxed area in $A$, showing only the primary afferents; the solid arrow indicates the injected afferent, and arrowheads indicate the coupled afferents, some of which are only weakly filled and partially overlap other filled afferents. The coupling site with LG (open arrow) is proximal to the coupling site between primary afferents (open arrowhead). $C$, Orthodromic transmission between a primary afferent $\left(1^{\circ} A\right)$ (spike evoked by injection of depolarizing current) and LG. $D$, Mutual synaptic interactions between primary afferent axons. Depolarizing current injection into $1^{\circ} \mathrm{A}$ (2) evokes a depolarization and train of spikes that produce a smaller depolarization and corresponding EPSPs in $1^{\circ} \mathrm{A}(1)$.
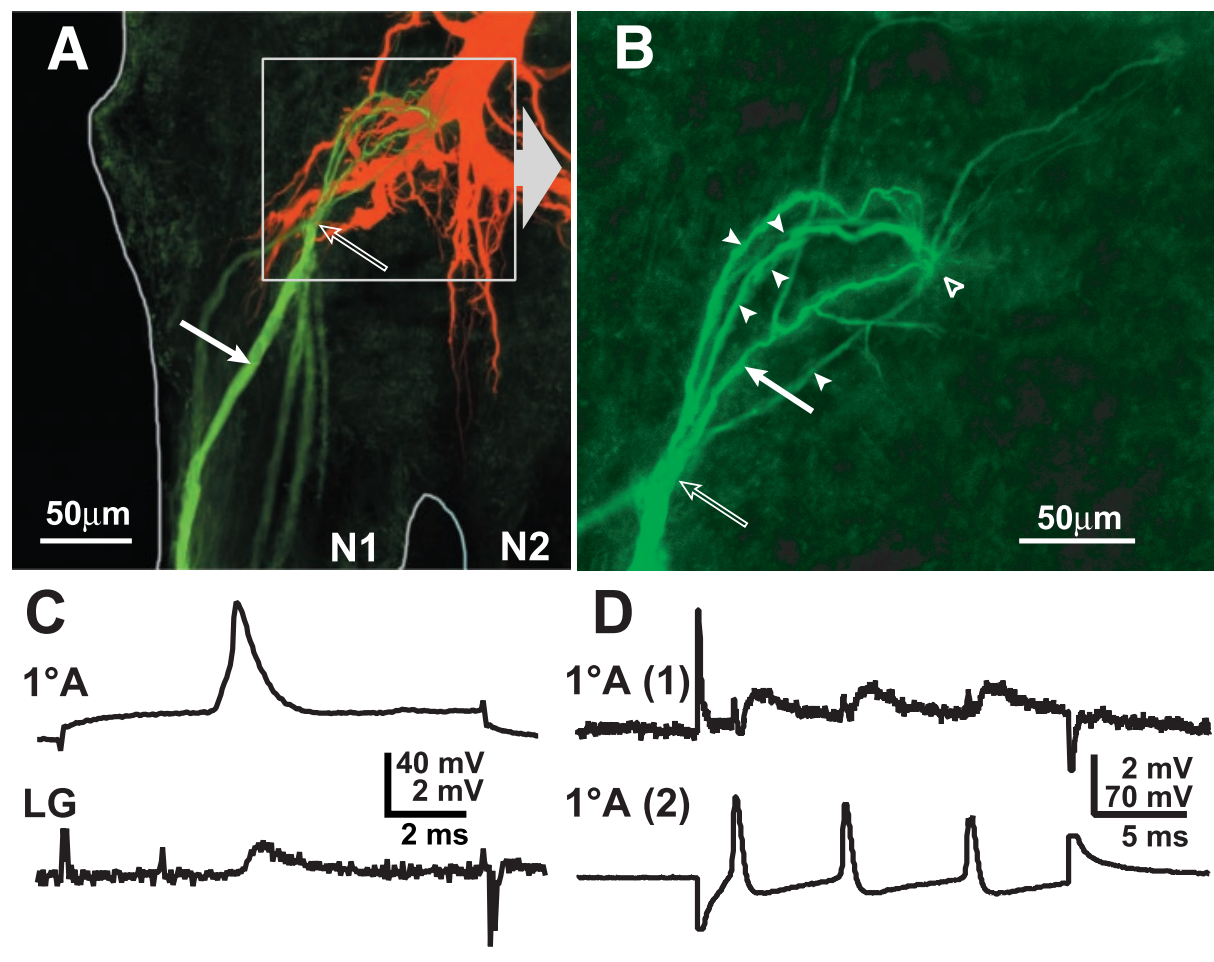

1991). An antidromically activated LG spike evokes a small antidromic synaptic potential in the primary afferent, consistent with current flow in both directions (data not shown).

Evidence for direct electrical and synaptic coupling between afferents in the same nerve that contact LG was obtained when depolarizing current injected into one afferent depolarized the cell and evoked a train of spikes and a corresponding smaller depolarization and train of EPSPs in a second afferent (Fig. 3D). A reciprocal response occurred when the same current was applied to the other cell (data not shown). LG did not fire in either case.

\section{Afferent recruitment by the lateral excitatory network}

Short-latency EPSPs also appeared in an afferent axon in response to electrical stimulation of the sensory nerve containing the axon (Fig. 3). This occurred when the stimulus directly excited the axons of afferents that presumably were coupled to the recorded afferent. Increased stimulus intensities recruited larger EPSPs in the recorded afferent and in LG (Fig. 4A). The EPSP increases in afferents were discontinuous with the rise in stimulus voltage, as though single elements were recruited at discrete stimulus thresholds. Up to six such distinguishable EPSP levels occurred in each recorded afferent before the afferent was stimulated directly by shock to the sensory nerve (Fig. 4A). The number of recorded EPSPs is dependent on the stimulus threshold of the recorded afferent relative to those of the afferents coupled to it; threshold is influenced in part by inherent properties of the afferents concerned as well as the relative position of the stimulating electrode. The number of recorded EPSPs therefore differed from the anatomical results. In the example of Figure $4 B$, a smaller stimulus (light gray) evoked EPSPs in both the primary afferent and LG, and a larger stimulus (dark gray) evoked a larger afferent EPSP that brought the cell to threshold. The appearance of the recruited afferent action potential coincided with an incremental increase in the compound EPSP in LG. A further increase in the nerve stimulus (black) evoked the afferent spike directly and so shortened the latencies of both the

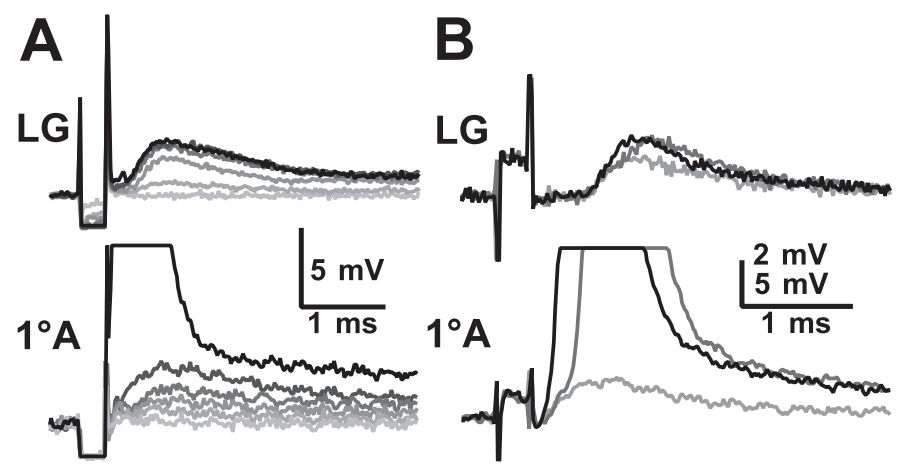

Figure 4. Responses of LG (top) and a $1^{\circ} \mathrm{A}$ (bottom) to electrical stimulation of the nerve containing the $1^{\circ} \mathrm{A}$ axon. $A$, Increasing stimulus amplitudes elicited several increasing EPSPs in LG and in the $1^{\circ} \mathrm{A}$ before reaching the stimulus threshold (black) of the $1^{\circ} \mathrm{A} . B$, Increasing stimulus amplitudes evoked an EPSP (light gray), a recruited $1^{\circ} \mathrm{A}$ spike (dark gray), and a directly evoked spike (black) in the $1^{\circ} \mathrm{A}$ and corresponding EPSPs in LG. $A, B$, Results from two different experiments.

afferent spike and the added EPSP in LG. Afferents that we could not demonstrate to contact LG, 12\% (14 of 120), still received EPSPs from other afferents.

\section{LG depolarization and afferent, interneuron recruitment}

The response of the afferent to synaptic input from other afferents could be modulated by changes in the membrane potential of LG (Fig. 5A). Sensory nerve stimuli that evoked a subthreshold EPSP in an afferent became superthreshold when the LG was depolarized by $\sim 15 \mathrm{mV}$ (between 10 and $20 \mathrm{mV}$ ) at the initial segment by direct current injection there. Conversely, larger sensory nerve stimuli that evoked superthreshold EPSPs in the afferent when LG was at rest potential were made subthreshold by hyperpolarization of LG (Fig. 5B). The spread of current from LG into the afferent (causing a potential change of up to $\pm 1 \mathrm{mV}$ ) was apparent in recordings made from sites near the LG-afferent 


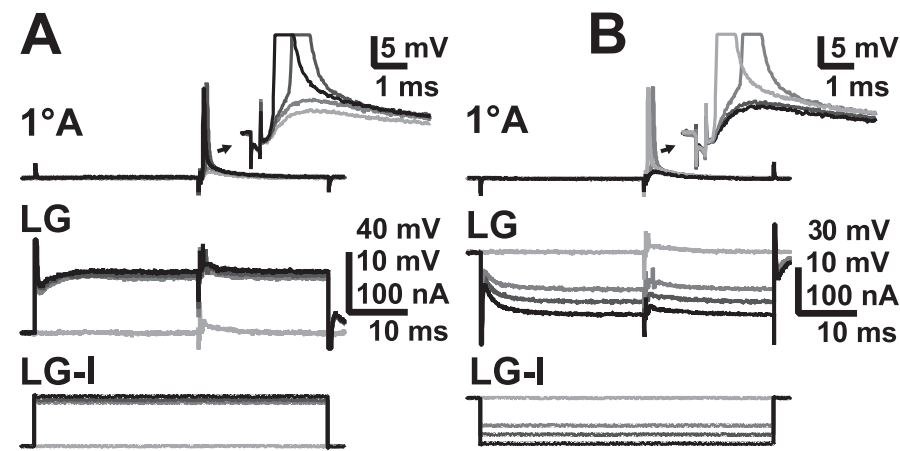

Figure 5. Effect of LG depolarization and hyperpolarization on recruitment of primary afferents. $A$, Response of a $1^{\circ} \mathrm{A}$ (top traces and inset) to a consistent subthreshold sensory nerve stimulation when LG (middle traces) is at rest (light gray) and when LG is increasingly depolarized (darker grays, then black) by injected current (LG-I, bottom traces). B, Response of a $1^{\circ} \mathrm{A}$ (top traces and inset) to normally suprathreshold sensory nerve stimulation when LG (middle traces) is at rest (light gray) and when LG is increasingly hyperpolarized (darker grays, then black) by injected current (LG-I, bottom traces).

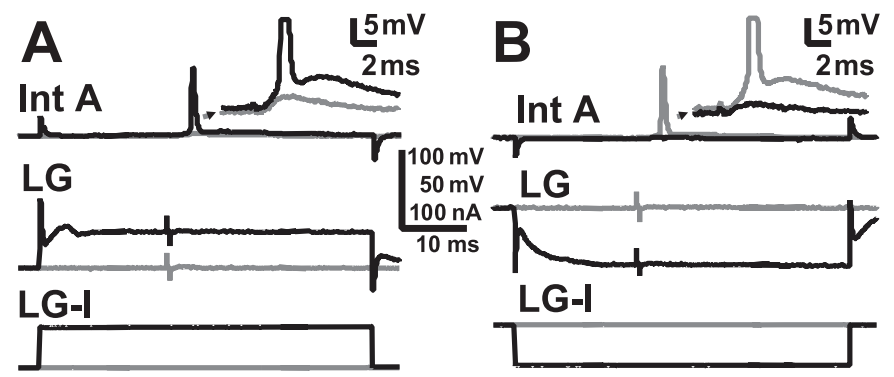

Figure 6. Effect of LG depolarization and hyperpolarization on interneuron A recruitment. $A$, Response of Int A (top and inset) to normally subthreshold sensory nerve stimulation when LG (middle traces) is at rest ( gray) and when LG is depolarized (black) by injected current (LG-I, bottom traces). B, Response of Int A (top traces and inset) to normally suprathreshold sensory nerve stimulation when LG (middle traces) is at rest (gray) and when LG is hyperpolarized (black) by injected current (LG-I, bottom traces).

contact point (data not shown); the very small potential changes shown in Figure 5 were recorded from a site distant from LG.

Depolarization of LG by a similar amount also helped recruit first-order mechanosensory interneurons that excite LG, including interneuron A (Int A, also known as 6B1) (Sigvardt et al., 1982; Wine, 1984; Nagayama et al., 1997). Depolarization of LG made subthreshold inputs to first-order interneurons $(N=17$; eight of which were identified as Int A) become suprathreshold (Fig. 6A). Conversely, hyperpolarization of LG caused a suprathreshold sensory nerve stimulus to Int A to become subthreshold (Fig. 6B). Although Int A had been thought not to excite LG in the terminal ganglion (Zucker, 1972), we found that Int A was electrically coupled to LG there as in more rostral abdominal ganglia (Edwards et al., 1991). Current injection into LG had a measurable effect on the membrane potential of Int A, and directly evoked spikes in Int A produced small, short-latency EPSPs in the A6 LG. The effects of LG depolarization on excitation of Int A, and other interneurons as well, can therefore be mediated through the recruitment of primary afferents that excite them and more directly through antidromic current flow from LG into these neurons.

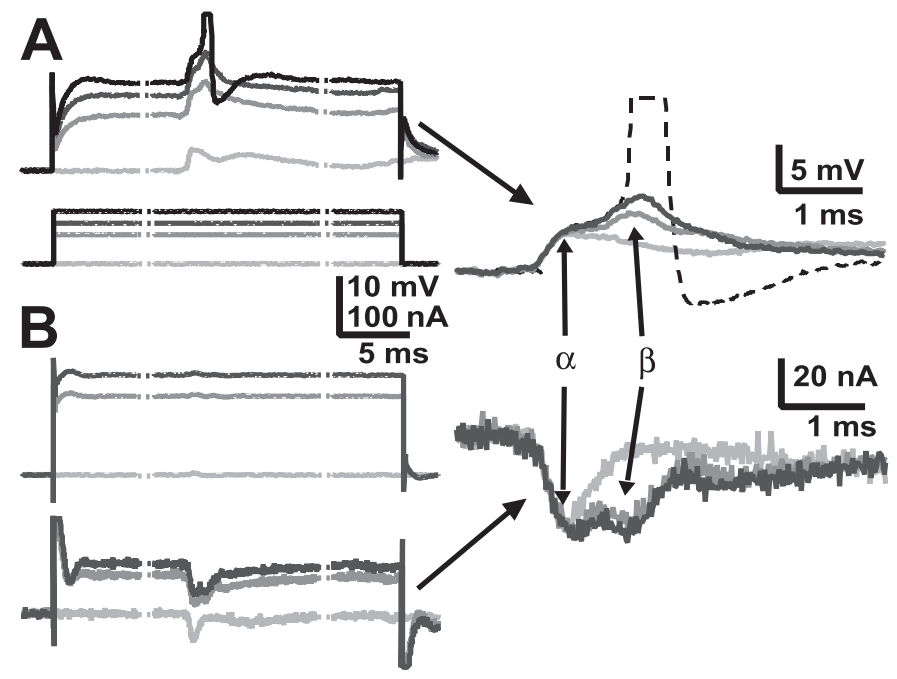

Figure 7. Modulation of LG synaptic inputs by LG depolarization. $A$, EPSPs in LG (top) evoked by nerve stimulation when LG was at rest or depolarized by current injection (bottom). B, EPSCs in the same LG (bottom) evoked by the same nerve stimulation when LG was voltage clamped to rest potential and to depolarized levels (top). Right, Baseline corrected voltage responses (top) and current responses (bottom). The $\alpha$ and $\beta$-components of each are identified. The stippled lines indicate breaks in the traces; the actual duration of current injection was $50 \mathrm{msec}$.

\section{LG depolarization and LG recruitment}

Finally, the same depolarization of LG that helped a sensory nerve shock recruit afferents and first-order interneurons also increased synaptic inputs to LG and brought the cell to threshold (Fig. 7). The $\beta$ component of the LG EPSP, which results from interneuron inputs, increased when LG was depolarized by injected current (Fig. 7A) and, with sufficient current, brought LG to threshold. The depolarizing current increases the excitability of LG (note the delayed rectification in the LG response to the current), and it recruits additional inputs, particularly from interneurons, to LG. The evidence of these inputs was obtained when LG responses evoked by the same stimuli were measured under two-electrode voltage clamp and the synaptic currents produced by primary interneurons also increased (Fig. 7B).

\section{A model of the lateral excitatory network}

The operation of the network depends on simultaneous current flows between primary afferents, LG, and interneurons. To understand these interactions, we constructed a multicompartment model of the network that represents the basic pattern of neuronal connectivity and synaptic properties in the network, but in the interest of simplicity ignores cellular anatomy and much biophysical detail. The model network consists of an array of 10 single model compartments that represent primary afferents, two single compartment model interneurons, and a two-compartment model LG (Fig. 8). The primary afferent models are electrically coupled through 6.25 $\mathrm{M} \Omega$ resistances in the pattern displayed in Figure $8 A$. This pattern enables some afferents to be connected to two others, others to three, and still others to four other afferents. Each model interneuron is excited through excitatory chemical synapses by six afferents; two of the afferents excite both interneurons. LG is excited by all of the afferents through rectifying electrical synapses that converge on the passive LG dendritic compartment $\left(\mathrm{LG}_{\mathrm{D}}\right)$, and by the interneurons, which make identical rectifying electrical synapses on the $\mathrm{LG}$ initial segment compartment $\left(\mathrm{LG}_{\mathrm{IS}}\right) . \mathrm{LG}_{\mathrm{D}}$ is linked by a $0.5 \mathrm{M} \Omega$ coupling resis- 
A

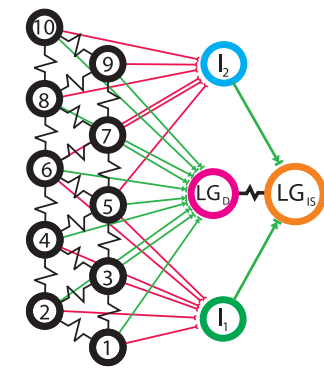

Recruited Afferent, with the order of recruitment

Recruited interneuron or LG

Stimulated Afferent

OUnstimulated Afferent
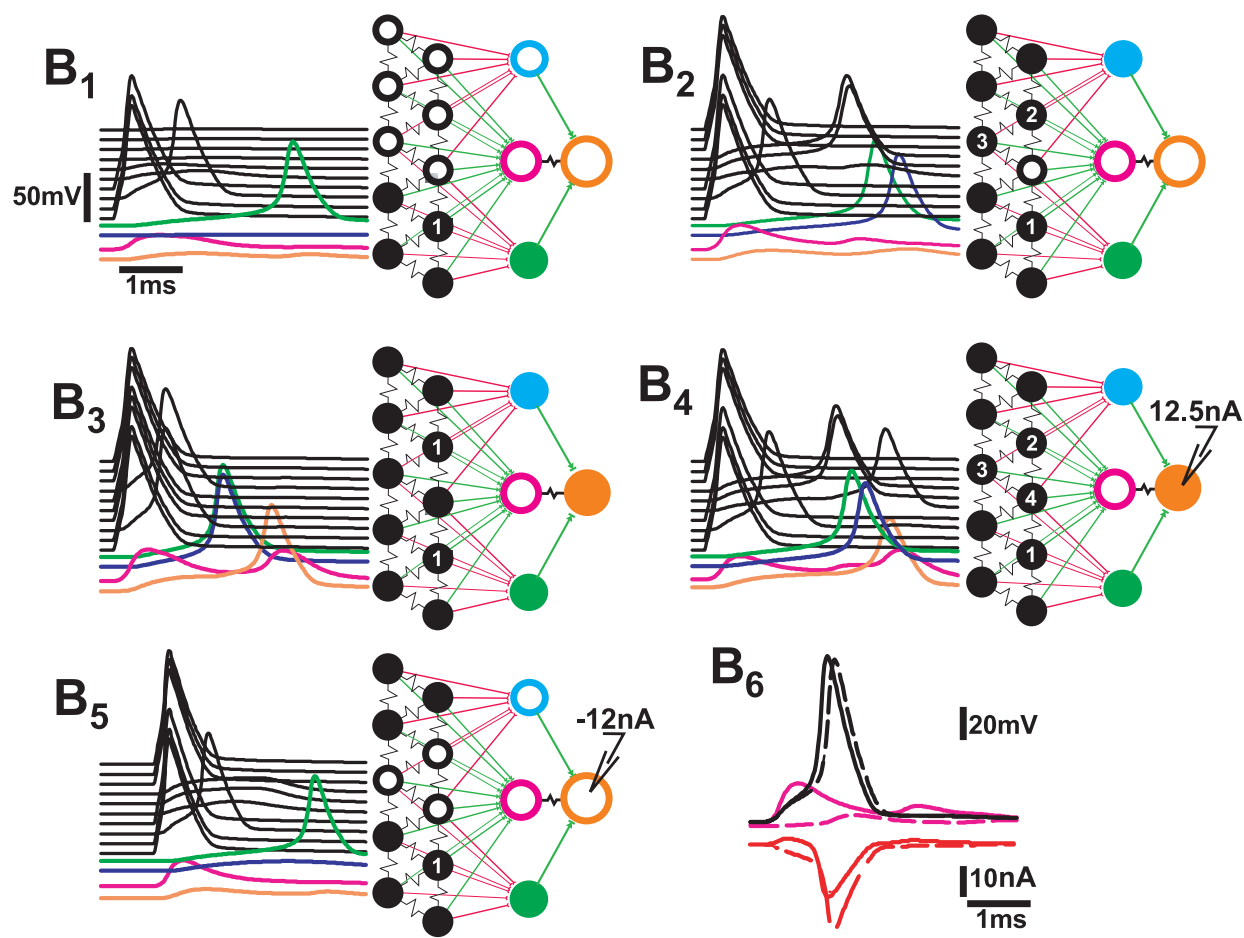

Figure 8. Electrical circuit model of the lateral excitatory network and LG. $A$, Each model compartment was characterized by reversal potentials and maximal conductances for sodium, potassium, and leakage currents. In all the compartments except $\mathrm{LG}_{\mathrm{D}}$, which was passive, the sodium and potassium conductances were voltage- and time-dependent, as described by Hodgkin and Huxley (1952). B, Responses of the network to patterns of afferent stimulation. The $5 \mathrm{msec}$ time course of each compartmental response is at left in each panel; interneuron and LG compartment responses are shown color-coded at the bottom; afferent responses are black and are arranged in ascending order according to the diagram in $A$. The pattern of stimulation and the network response are shown in the diagram at right in each panel, where brighter colored compartments are those that produced a spike. $B_{1}$, Responses to simultaneous stimulation of compartments 1,2 , and 4 with $0.3 \mathrm{msec}$ pulses of $300 \mathrm{nA}$ depolarizing current. $B_{2}$, Responses to stimulation of afferent compartments $1,2,4,8,9,10 . B_{3}$, Stimulation of all afferents except 3 and 7 . $B_{4}$, Afferents were stimulated according to the pattern used in $B_{2} 10 \mathrm{msec}$ after onset of $12.5 \mathrm{nA}$ current injection into $\mathrm{LG}_{\mathrm{IS}} . B_{5}$, Afferents were stimulated according to the pattern of $B_{2} 10 \mathrm{msec}$ after onset of -12 $\mathrm{nA}$ current injection into $\mathrm{LG}_{\mathrm{IS}}$. $B_{6}$, The effect of the $\mathrm{LG}_{\mathrm{D}}$ EPSP on afferent recruitment. Afferents were stimulated according to the pattern of $\mathrm{B}_{2}$ when the network was intact (continuous traces) and when all afferent synapses to $\mathrm{LG}_{\mathrm{D}}$ except that of afferent 5 were removed (dashed traces). Top, Voltage responses (in millivolts) of afferent 5 and $\mathrm{LG}_{\mathrm{D}}$ Bottom, Transynaptic current (in nanoamperes) through the rectifying electrical synapse between afferent 5 and $L_{\mathrm{D}}$. Positive current moves from $\mathrm{LG}_{\mathrm{D}}$ to afferent 5.

tance to $\mathrm{LG}_{\mathrm{IS}}$. The electrical properties of the model compartments, chemical and electrical synapses, and the integration technique used to calculate compartmental voltages and currents are all described in Material and Methods. All compartments of the model are assumed to support Hodgkin-Huxley-like active membrane currents (Hodgkin and Huxley, 1952) except the LG dendrite compartment, which is passive, reflecting the passive behavior of the LG dendrites (Edwards et al., 1994). The ionic equilibrium potentials were adjusted to produce resting potentials in the three types of model neurons that reproduced the differences in the resting membrane potentials recorded from the three neurons (Heitler et al., 1991). The electrical properties of the different compartments were chosen to produce measures of input resistance and current threshold similar to what was recorded experimentally. Similarly, parameters that govern chemical and synaptic conductances were chosen to reproduce the synaptic and coupling responses recorded from these neurons.

To simulate the afferent responses to sensory nerve shock, depolarizing current pulses $(300 \mathrm{nA}, 0.3 \mathrm{msec})$ were applied simultaneously to individual afferent compartments. Simultaneous stimulation of three model afferents at one end of the array led to the recruitment of an adjacent afferent and an interneuron that was excited by all four afferents (Fig. $8 B_{1}$ ). LG responded with small EPSPs in both the dendritic and initial segment com- partments. Responses to increased stimulus strength were simulated by adding three more afferents at the other end of the array to those receiving simultaneous stimulation. Stimulation of these six afferents caused two more afferents to be recruited, followed by both interneurons (Fig. $8 B_{2}$ ). The responses of LG were again below threshold. When two more afferents were added to the group of six receiving simultaneous stimulation, the remaining two afferents were recruited at short latency, followed by the interneurons and then LG (Fig. $8 B_{3}$ ). LG could also be brought to threshold if the previous pattern of stimulation of six afferents (Fig. $8 B_{2}$ ) occurred during a period of depolarization induced by injection of a constant $12.5 \mathrm{nA}$ of current into the initial segment compartment (Fig. 8B $B_{4}$ ). The depolarizing current caused the last afferent to be recruited and it caused each of the recruited afferents and interneurons to fire earlier. The earlier and greater synaptic inputs and the increase in the excitability produced by the direct depolarization of LG were sufficient to bring LG to threshold. Finally, the recruitment of afferents and interneurons was reduced when the afferent stimulation occurred while the LG was hyperpolarized by injection of $-12 \mathrm{nA}\left(\right.$ Fig. $8 B_{5}$ ). The stimulus pattern, which had recruited three additional afferents and both interneurons (Fig. $8 B_{2}$ ), only recruited one afferent and one interneuron when the stimulus was presented during the hyperpolarization of LG. 
As in the experimental preparations, recruitment of unstimulated afferents depends on the coupling between them and the stimulated afferents and on antidromic current from the LG dendrites into the unstimulated afferents. The importance of the antidromic current in this simulation is shown in Figure $8 B_{6}$, in which the stimulus pattern of Figure $8 B_{2}$ was repeated, first under the original conditions (continuous lines), and second when the rectifying electrical synapses between all but one of the afferents and the $\mathrm{LG}_{\mathrm{D}}$ compartment were removed (dashed lines). In the first case, the converging inputs from the stimulated afferents evoked a fast-rising EPSP (pink continuous trace) in $\mathrm{LG}_{\mathrm{D}}$ that exceeded the smaller EPSP in the unstimulated afferent (black continuous trace). As a result, the current through the rectifying electrical synapse that connects the unstimulated afferent and $\mathrm{LG}_{\mathrm{D}}$ compartments was initially positive, in the antidromic direction from $\mathrm{LG}_{\mathrm{D}}$ to the afferent (red continuous line). This current contributed to the afferent EPSP and helped bring the afferent to threshold. The resulting afferent spike then produced a much larger orthodromic (i.e., negative in Fig. $8 B_{6}$ ) synaptic current that contributed to the EPSP of the LG. In the second simulation, the EPSP in $\mathrm{LG}_{\mathrm{D}}$ (pink dashed line in the top panel) was made much smaller by removing the synapses to LG from all but the recorded afferent. As a result, the larger afferent EPSP drove current in the orthodromic direction (red dashed line, bottom panel), and so transferred charge from the afferent to $\mathrm{LG}_{\mathrm{D}}$. As a result, the afferent EPSP (dashed black trace in the top panel) reached threshold later than when the full LG EPSP occurred.

Recruitment of afferents is also facilitated by the rise in resistance presented by the rectifying electrical synapses when the LG response exceeds the afferent response. In the present model, the resistance of the synapses at rest was $5.4 \mathrm{M} \Omega$; when the difference between the afferent and LG potential was greatest in the reverse direction $(9.5 \mathrm{mV})$, the synaptic resistance would be nearly three times greater, if it were allowed to change instantaneously with the transynaptic potential. However, we have chosen to let the kinetics of the model rectifying electrical synapse be governed by a time constant of $0.75 \mathrm{msec}$, the value obtained from kinetic measurements of the rectifying electrical giant motor synapse (Jaslove and Brink, 1986). As a result, the synaptic resistance increase was less, to $\sim 7 \mathrm{M} \Omega$. Nonetheless, this added synaptic resistance should increase the input resistance of the afferent and enable the coupling currents that it receives from neighboring afferents to have greater depolarizing effect. Simulations in which the synaptic resistance was allowed to change instantaneously with the voltage difference across the synapse, and so to achieve much higher values, caused less antidromic current and more orthodromic synaptic current to flow (data not shown). This altered pattern of synaptic currents caused the initial LG EPSP to be larger but the recruitment of unstimulated afferents to be reduced.

\section{DISCUSSION}

\section{The lateral excitatory network}

Earlier work demonstrated that the LG neuron is both directly excited by an array of primary mechanosensory afferents and indirectly excited by those same afferents through a set of mechanosensory interneurons that contact LG (for review, see Edwards et al., 1999). We have modified this description by showing that excitation of LG in the terminal abdominal ganglion is enhanced by a set of lateral excitatory networks among the primary afferents. Each lateral excitatory network is formed primarily among groups of afferent axons that innervate a common portion of the tailfan; these afferents project centrally through one of the sixth ganglionic nerves to contact first-order mechanosensory interneurons and LG (Newland et al., 2001). In addition, we found that depolarization of LG enhances recruitment of unstimulated afferents and interneurons, suggesting that the synaptic response of LG works to enhance the inputs of the cell itself. In nature, these network interactions and the interactions between LG and its presynaptic afferents and interneurons should help determine whether LG will respond and the crayfish will escape to a phasic mechanosensory stimulus.

The coupling between afferents is mediated by what appear to be nonrectifying electrical synapses. Because the coupling is primarily confined to afferents within single nerves, it acts to amplify stimuli that affect discrete areas of the tailfan innervated by those afferents. Recruitment of unstimulated afferents requires stimulation of a critical density of afferents to provide sufficient convergent, synchronous inputs to coupled unstimulated afferents. These criteria being met would lead in turn to excitation of other afferents and first-order mechanosensory interneurons. Such a chain reaction mechanism could amplify responses to stimuli above a critical intensity by recruiting a large fraction of the coupled afferents and first-order interneurons.

Similar electrical coupling occurs among retinal cones (Hsu et al., 2000) and among proprioceptive afferents in the walking legs of crayfish (El Manira et al., 1993). However, because the responses of cones to light is a graded hyperpolarization, spread of this potential to neighboring cones would not contribute to a chain reaction among primary afferents of the type described here. In contrast, the coupling among crayfish proprioceptive afferents may serve as a mechanism to amplify proprioceptive input.

Additionally, synaptic coupling among neighboring afferents of the excitatory neurons of single cortical barrels in the mouse, each of which receives projections from a single whisker, forms an excitatory network that is primarily confined to the barrel (Petersen and Sakmann, 2000). The function of this network is unknown, but may be to amplify inputs to the barrel.

\section{Feedback modulation by LG}

The ability of the membrane potential of LG to modulate the excitability of the afferents and primary interneurons creates a positive feedback that amplifies the lateral excitation. Although we have demonstrated this effect by depolarizing LG with injected current, the natural source of depolarization is the EPSP produced in the LG dendrite by the same set of stimulated afferents. In this mechanism, the LG EPSPs would amplify themselves by helping to recruit additional afferents that excite LG.

Two mechanisms may allow LG depolarization to increase the excitability of presynaptic primary afferents. The first is the flow of antidromic current that depolarizes the afferent axon. Unlike the tonic depolarization of LG imposed by injected current, dendritic LG EPSPs are both large and brief, and the expected antidromic currents should have the same character. Such phasic antidromic currents should have a greater effect on the excitability of the afferent axon than did the tonic currents produced by imposed depolarization of LG (Fig. 5). The simulations of Figure $8 B_{6}$ show that the antidromic synaptic current can advance the recruitment of afferents; larger dendritic EPSPs would presumably have greater effects. The EPSPs in the $\mathrm{LG}_{\mathrm{D}}$ compartment were $25 \mathrm{mV}$ in amplitude; larger EPSPs have been recorded in LG dendrites (Zucker, 1972), and so should have correspondingly 
greater effects in recruiting unstimulated afferents. A mechanism like this appears to operate in the Mauthner neuron (the M-cell) of teleost fish, where EPSPs induce an antidromic junctional current that excites the axon terminals of primary afferents that are electrically coupled to the M-cell dendrite. The afferent spike then drives additional orthodromic synaptic current into the M-cell dendrite to enhance the EPSP (Pereda et al., 1995). Afferents recruited by an LG EPSP would be expected to do the same.

The second mechanism results from the rectifying nature of the synapses that link the afferents to LG (Edwards et al., 1991, 1998). This rectification causes the transynaptic conductance to be reduced when the LG is depolarized relative to the afferent and to be increased when the reverse is true. The LG EPSPs should reduce the conductance of synapses from unstimulated afferents onto LG, increasing the local input resistance of the afferent axon in the region where coupling currents are integrated. As a result, inputs from other coupled afferents will be more effective in exciting the axon.

The balance of these effects depends on the nature of the rectifying electrical synapses between the afferents and LG. Formal analysis of these synapses is made difficult by the remoteness of recording and current injection sites from the synapses in both presynaptic and postsynaptic cells. Our results suggest, however, that significant differences exist between these synapses and the giant motor synapse (GMS) that LG makes with the giant motor neuron. Unlike the GMS, where the resting transynaptic conductance is low, here between the afferents and LG it appears to be high. Hyperpolarizing current is readily passed antidromically, and depolarizing current can also pass, although not as easily (J. Herberholz and D. Edwards, unpublished observations). Although our model rectifying electrical synapses are based on descriptions of the GMS (Jaslove and Brink, 1986; Giaume et al., 1987), we have modified the voltage threshold to reflect this difference.

From these results and examples, it is apparent that a lateral excitatory network creates a chain reaction that helps amplify inputs and discriminate signal from noise. We have shown that the density of primary afferents that are excited within one sensory nerve is critical for further activation of the network by the membrane potential of LG and for the subsequent amplification of sensory inputs onto LG. The network is tuned to amplify only strong inputs that activate a high number of afferents and to discriminate against weak inputs. In this way, the network, with the integral participation of LG, would act as a coincidence detector for activity within a local population of neurons. The selective responsiveness to phasic stimulation of LG is conditional on its short time constant. Activation of the network provides amplification and synchronization of such sensory inputs that occur within a brief period of time.

The largest effect of both the lateral excitatory network and its modulation by LG EPSPs may be on the recruitment of mechanosensory interneurons that drive the decisive $\beta$ component of the LG EPSP. We have shown here how the same LG depolarization that helps recruit unstimulated afferents also helps recruit Int $\mathrm{A}$ and other unidentified primary mechanosensory interneurons and increase the $\beta$ component of the LG synaptic current. The effect of the depolarization of LG on the recruitment of primary interneurons is twofold: first, additional primary afferents are recruited and create increased inputs onto LG and primary interneurons. Second, the excitability of primary interneurons is enhanced by antidromic current flow from LG back into these cells. The significance of each of these mechanisms for LG excitation depends on the pattern of sensory nerve stimulation (Herberholz and Edwards, unpublished observations).

\section{The escape command}

Previous work suggested that the decision mechanism lay exclusively in the LG, where converging inputs created dendritic currents that converged on the spike initiation zone (Olson and Krasne, 1981). Our results indicate that part of the decision mechanism lies in the interaction between LG and the lateral excitatory network. The afferent recruitment mediated by the lateral excitatory network is a form of nonlinear amplification that helps the escape circuit identify the patterns and levels of input that should trigger an escape response. This nonlinear amplification should reduce the stimulus threshold for escape, and it should make the threshold more discrete. Finally, the network provides many targets for modulation. Serotonin, for example, has been shown to alter the stimulus threshold of LG in ways that depend on the pattern of serotonin exposure (Teshiba et al., 2001); some of these effects may result from changes in the excitability of the lateral excitatory network.

\section{REFERENCES}

Antonsen BL, Edwards DH (2000) Activity-dependent dye-coupling in the lateral giant circuit of crayfish. Soc Neurosci Abstr 26:1725.

Burrows M, Matheson T (1994) A presynaptic gain control mechanism among sensory neurons of a locust leg proprioceptor. J Neurosci 14:272-282.

Calabrese RL (1976) Crayfish mechanoreceptive interneurons. Part 1. The nature of ipsilateral excitatory inputs. J Comp Physiol [A] 105:83-102.

Clarac F, Cattaert D, Le Ray D (2000) Central control components of a "simple" stretch reflex. Trends Neurosci 23:199-208.

Edwards DH, Heitler WJ, Leise EM, Fricke RA (1991) Postsynaptic modulation of rectifying electrical synaptic inputs to the LG escape command neuron in crayfish. J Neurosci 11:2117-2129.

Edwards DH, Yeh SR, Barnett LD, Nagappan PR (1994) Changes in synaptic integration during the growth of the lateral giant neuron of crayfish. J Neurophysiol 72:899-908.

Edwards DH, Yeh SR, Krasne FB (1998) Neuronal coincidence detection by voltage-sensitive electrical synapses. Proc Natl Acad Sci USA 95:7145-7150.

Edwards DH, Heitler WJ, Krasne FB (1999) 50 years of a command neuron: the neurobiology of escape behavior in the crayfish. Trends Neurosci 22:153-161.

El Manira A, Cattaert D, Wallen P, DiCaprio RA, Clarac F (1993) Electrical coupling of mechanoreceptor afferents in the crayfish: a possible mechanism for enhancement of sensory signal transmission. J Neurophysiol 69:2248-2251.

Giaume C, Kado RT, Korn H (1987) Voltage-clamp analysis of a crayfish rectifying synapse. J Physiol (Lond) 386:91-112.

Hartline HK, Ratliff F (1957) Inhibitory interaction of receptor units in the eye of Limulus. J Gen Physiol 40:1357-1376.

Heitler WJ, Fraser K, Edwards DH (1991) Different types of rectification at electrical synapses made by a single crayfish neurone investigated experimentally and by computer simulation. J Comp Physiol [A] 169:707-718

Hodgkin AL, Huxley AF (1952) A quantitative description of membrane current and its application to conduction and excitation in nerve. J Physiol (Lond) 117:500-544.

Hsu A, Smith RG, Buchsbaum G, Sterling P (2000) Cost of cone coupling to trichromacy in primate fovea. J Opt Soc Am A Opt Image Sci Vis 17:635-640.

Jaslove SW, Brink PR (1986) The mechanism of rectification at the electrotonic motor giant synapse of the crayfish. Nature 323:63-65.

Krasne FB (1969) Excitation and habituation of the crayfish escape reflex: the depolarizing response in lateral giant fibres of the isolated abdomen. J Exp Biol 50:29-46.

Nagayama T, Aonuma H, Newland PL (1997) Convergent chemical and electrical synaptic inputs from proprioceptive afferents onto an identified intersegmental interneuron in the crayfish. J Neurophysiol $77: 2826-2830$. 
Newland PL, Aonuma H, Nagayama T (2001) The role of proprioceptive signals in the crayfish escape circuit. Zool Sci (Tokyo) 17:1185-1195.

Olson GC, Krasne FB (1981) The crayfish lateral giants as command neurons for escape behavior. Brain Res 214:89-100.

Pereda AE, Bell TD, Faber DS (1995) Retrograde synaptic communication via gap junctions coupling auditory afferents to the Mauthner cell. J Neurosci 15:5943-5955.

Petersen CC, Sakmann B (2000) The excitatory neuronal network of rat layer 4 barrel cortex. J Neurosci 20:7579-7586.

Shepherd GM, Brayton RK (1979) Computer simulation of a dendrodentritic synaptic circuit for self- and lateral-inhibition in the olfactory bulb. Brain Res 175:377-382.

Sigvardt KA, Hagiwara G, Wine JJ (1982) Mechanosensory integration in the crayfish abdominal nervous system: structural and physiological differences between interneurons with single and multiple spike initiating sites. J Comp Physiol 148:143-157.

Teshiba TM, Shamasian A, Yashar B, Yeh SR, Edwards DH, Krasne FB (2001) Dual and opposing modulatory effects of serotonin on crayfish lateral giant escape command neurons. J Neurosci 21:4523-4529.

Wiese K (1976) Mechanoreceptors for near-field water displacements in crayfish. J Neurophysiol 39:816-833.

Wine JJ (1984) The structural basis of an innate behavioural pattern. J Exp Biol 112:283-319.

Zucker RS (1972) Crayfish escape behavior and central synapses. I. Neural circuit exciting lateral giant fiber. J Neurophysiol 35: $599-620$. 